\title{
HIDING THE VECTOR RESONANCE SIGNAL
}

We investigate how the interplay of the direct and mixing-induced couplings of the hypothetical $S U(2)_{L+R}$ vector triplet resonance to the third quark generation can hide the resonance signal in $\bar{t} t, \bar{b} b$, and $\bar{b} t / \bar{t} b$ channels. The vector resonances are assumed to represent bound states of hypothetical strongly-interacting new physics, the extension of the Standard model.

Keywords: Electroweak symmetry breaking, effective Lagrangian, vector resonances, new strong interactions.

\section{Introduction}

Even though the ATLAS and CMS announcements of the $125 \mathrm{GeV}$ boson discovery [1] have not settled the question about the mechanism of electroweak symmetry breaking yet they did provide major hints pointing to its solution. At the moment, it is clear that the observed properties of the discovered boson are compatible with the Standard model Higgs boson hypothesis [2 and 3]. At the same time they are compatible with many alternative extensions of the Standard model (SM). From a theoretical point of view, the extensions get some preference over the SM Higgs due to the naturalness argument. They include supersymmetry theories as well as theories where electroweak symmetry is broken by new strong interactions, like in Technicolor [4 - 7].

Following theoretical arguments, as well as the example of QCD, it seems reasonable to expect that beside the composite scalar the new strong interactions would also produce bound states of higher spins. The vector $S U(2)$ triplet resonance is a natural candidate to look for. From other point of view, in strongly interacting theories new resonances are required to tame the unitarity. If, as expected, the composite Higgs couplings differ from the SM ones the Higgs alone will fail to unitarize the $V V\left(V=W^{ \pm}, Z\right)$ scattering amplitudes and other resonances are necessary to do the job.

Recently, we studied the effective Lagrangian where beside the $125-\mathrm{GeV}$ scalar resonance - an $S U(2)_{L+R}$ singlet complementing the non-linear triplet of the Nambu-Goldstone bosons, the $S U(2)_{L+R}$ triplet of vector resonances is explicitly present. It fits the situation when the global $S U(2)_{L} \times S U(2)_{R}$ symmetry is broken down to $S U(2)_{L^{+} R^{*}}$ As far as the vector resonance sector is concerned the vector triplet is introduced as a gauge field via the hidden local symmetry approach [8]. Because of this, the vector resonance mixes with the EW gauge bosons which result in appearance of indirect couplings of the vector resonance with all SM fermions. Besides, the direct couplings of the vector resonance triplet to the SM fermions are also allowed by the Lagrangian symmetry. Regarding the direct couplings we opt for a special setup inspired by the speculations about an extraordinary role of the top quark (or the third quark generation) in new strong physics: we admit direct couplings of the new triplet to no other SM fermions but the top and bottom quarks only. Finally, the symmetry allowed interaction terms between the scalar and vector resonances are also present. We nicknamed the effective Lagrangian as the tBESS model.

In the paper [9], we had introduced the so-called Death Valley (DV) effect. The DV is a region in the $\left(b_{L}, b_{R}\right)$ parameter space where the interplay of the direct and indirect couplings of the vector triplet with fermions can diminish or even zero a particular top/bottom quark channel decay width of the vector resonance.

In this paper, we analyze the DV effect in the light of the updated low-energy limits for the parameters of the tBESS Lagrangian. The Higgsless tBESS model considered in [9] was made obsolete by the LHC discovery of the $125 \mathrm{GeV}$ boson. Following this development we augmented our effective Lagrangian with the scalar resonance representing the discovered boson and recalculated the low-energy limits [10 and 11]. In this paper we calculate the relevant DV regions and superimpose them over the regions allowed by the low-energy data.

In Section 2 we briefly explain the structure of the tBESS effective Lagrangian. In Section 3 we outline the structure of the low-energy limit of the tBESS Lagrangian and how the lowenergy fits and confidence level limits on its free parameters were obtained. The fits and limits are briefly summarized. Eventually, we present the obtained Death Valley regions in the superposition with the low-energy limits for the free parameters. In Section 4 we discuss our findings and conclusions.

\footnotetext{
* ${ }^{12}$ Mikulas Gintner, ${ }^{23}$ Josef Juran

'Physics Department, University of Zilina, Zilina, Slovakia, E-mail: gintner@fyzika.uniza.sk

${ }^{2}$ Institute of Experimental and Applied Physics, Czech Technical University in Prague, Prague, Czech Republic, E-mail: josef.juran@utef.cvut.cz

${ }^{3}$ Institute of Physics, Silesian University in Opava, Opava, Czech Republic
} 


\section{The effective Lagrangian}

We introduced the $S U(2)_{L+R}$ triplet vector resonance to the usual $S U(2)_{L} \times S U(2)_{R} \rightarrow S U(2)_{L+R}$ effective Lagrangian with the non-linearly transforming $S U(2)_{L+R}$ triplet of the would-be NambuGoldstone bosons augmented with the $S U(2)_{L+R}$ singlet scalar resonance. For simplicity, we assumed the SM couplings of the scalar resonance only in our analysis. Recall that the current LHC measurements admit about $10 \%$ deviations from the SM for the $125 \mathrm{GeV}$ boson couplings.

The vector triplet is brought in as a gauge field via the hidden local symmetry (HLS) approach [8]. The effective Lagrangian is built to respect the global $S U(2)_{L} \times S U(2)_{R} \times U(1)_{B-L} \times S U(2)_{H L S}$ symmetry of which the $S U(2)_{L} \times U(1)_{Y} \times S U(2)_{H L S}$ subgroup is also a local symmetry. The $S U(2)_{H L S}$ symmetry is an auxiliary gauge symmetry invoked to accommodate the $S U(2)$ triplet of vector resonances. Beside the scalar singlet and the vector triplet, the effective Lagrangian is built out of the SM fields only.

Our effective Lagrangian can be split in three terms

$$
\mathcal{L}=\mathcal{L}_{G B}+\mathcal{L}_{E S B}+\mathcal{L}_{\text {ferm }}
$$

where $\mathcal{L}_{G B}$ describes the gauge boson sector including the $S U(2)_{H L S}$ triplet, $\mathcal{L}_{E S B}$ is the scalar sector responsible for spontaneous breaking of the electroweak and hidden local symmetries, and $\mathcal{L}_{\text {ferm }}$ is the fermion Lagrangian of the model. We will not show full Lagrangian (1) here. All details regarding the Lagrangian can be found in [10 and 11]. Out of its three terms we will briefly discuss the third term, $\mathcal{L}_{\text {ferm }}$. The fermion part of the Lagrangian is directly related to the effects analyzed in this paper.

As far as the fermion sector is concerned no new fermions beyond the SM were introduced in our Lagrangian. The fermion sector of the Lagrangian can be divided into three parts

$$
\mathcal{L}_{\text {ferm }}=\mathcal{L}_{\text {ferm }}^{S M}+\mathcal{L}_{\text {ferm }}^{\text {scalar }}+\mathcal{L}_{(t, b)}^{\text {tBESS }}
$$

where $\mathcal{L}_{\text {ferm }}^{S M}$ contains the SM interactions of fermions with the electroweak gauge bosons, $\mathcal{L}_{\text {ferm }}^{\text {scalar }}$ is about the interactions of the fermions with scalar fields and includes the fermion masses, and $\mathcal{L}_{(t, b)}^{t B E S S}$ describes the third quark generation direct interactions with the vector resonance. In addition, it contains symmetry allowed non-SM interactions of the third quark generation with the EW gauge bosons.

The vector resonance couples directly to the third quark generation only. The interactions of the left and right fields are proportional to $b_{L}$ and $b_{R}$, respectively. In addition, there is a free parameter $p$ which disentangles the right bottom coupling from the right top coupling. The assumption that the vector resonance interaction with the right bottom quark is weaker than the interaction with the right top quark corresponds to the expectation that $0 \leq p \leq 1$. While $p=1$ leaves the interactions equal, the $p=0$ turns off the right bottom quark interaction completely and maximally breaks the $S U(2)_{R}$ part of the Lagrangian symmetry down to $U(1)_{R 3}$. In addition the symmetry of the Lagrangian admits non-SM interaction of the fermions with the EW gauge bosons that we also include in $\mathcal{L}_{(t, b)}^{t B E S S}$ under the assumption that they apply to the third quark generation only. These interactions are proportional to the free parameters $\lambda_{L}$ and $\lambda_{R}$. In the unitary (physical) gauge where all six unphysical scalar fields are gauged away the new physics part of the $(t, b)$ Lagrangian assumes the form

$\mathcal{L}_{(t, b)}^{t B E S S}=i b_{L} \bar{\psi}_{L}(X-W) \psi_{L}+i b_{R} \bar{\psi}_{R} P\left(X-B^{R 3}\right) \times$ $P \psi_{R}+i \lambda_{L} \bar{\psi}_{L}\left(\not W-B^{R 3}\right) \psi_{L}+i \lambda_{R} \bar{\psi}_{R} P\left(\not W-B^{R 3}\right) P \psi_{R}$

where $W_{\mu}=i g W_{\mu}^{a} \tau^{a}, B^{R 3}=i g^{\prime} \not B \tau^{3}, V_{\mu}=i \frac{g^{\prime \prime}}{2} X_{\mu}^{a} \tau^{a}$ and the matrix $P=\operatorname{diag}(1, p)$ disentangles the direct interaction of the vector triplet with the right top quark from the interaction with the right bottom quark.

The masses of the vector triplet are set by the scale $v$ and depend on the three gauge couplings $g, g^{\prime}, g^{\prime \prime}$, and the free parameter $\alpha$. In the limit when $g$ and $g^{\prime}$ are negligible compared to $g$ " the masses of the neutral and charged resonances are degenerate, $M_{V}=\sqrt{\alpha} g^{\prime \prime} v / 2$. If higher order corrections in $g / g$ " are admitted a tiny mass splitting occurs such that $M_{V^{0}}>M_{V^{ \pm}}[9]$.

Once the gauge boson fields are expressed in the gauge boson mass basis the mixing generated interactions of the vector triplet with all fermions will emerge from the fermion Lagrangian $\mathcal{L}_{\text {ferm }}^{S M}$. However, these indirect interactions of the vector resonance with the light fermions will be suppressed by the mixing matrix elements proportional to $1 / g$ ".

\section{The low-energy limits vs the Death Valley effect}

If there is the tBESS vector resonance triplet we can learn about its parameters even before its discovery by measuring deviations of the known particle couplings from their SM values. For example, due to the mixing between the vector resonance and the EW gauge bosons the deviations from the SM values would be present in the couplings of the EW gauge bosons to the SM fermions. In this sense, in the case of our effective Lagrangian the most interesting vertices should be those of the top and bottom quarks: $W t b, Z b b$, and $Z t t$.

Unfortunately, the measurements of the $W t b$ and $Z t t$ vertices have been rather coarse so far [12]. On the other hand, the couplings of the light fermion vertices including $Z b b$ were measured at previous colliders, sometimes to a very high precision. We refer to these measurement as the low-energy measurements. While the LHC is capable to refine these measurements, and it has done so already, the existing improvement are not sufficient 
to compete with the low-energy restrictions on the tBESS parameters.

To confront the tBESS free parameters with the low-energy measurements performed at $O\left(10^{2}\right) \mathrm{GeV}$ we derive the low-energy (LE) Lagrangian by integrating out the vector resonance triplet the assumed mass of which is $O\left(10^{3}\right) \mathrm{GeV}$. It proceeds by taking the limit $M_{\text {triplet }} \rightarrow \infty$, while $g$ " is finite and fixed, and by substituting the vector resonance equation of motion (EofM) obtained under these conditions.

Beside other Lagrangian terms the EofM also modifies the $\mathcal{L}_{(t, b)}^{t B E S S}$ term in (2). Thus

$$
\mathcal{L}_{\text {ferm }}^{L E} \equiv \mathcal{L}_{\text {ferm }}^{S M M}+\mathcal{L}_{(t, b)}^{L E-t B E S S}+\mathcal{L}_{\text {ferm }}^{\text {sclar }}
$$

In the EW gauge boson mass eigenstate basis and after the proper renormalization the relevant parts of the $\mathcal{L}_{\text {ferm }}^{L E}$ can be expressed as

$$
\begin{aligned}
& \mathcal{L}_{\text {ferm }}^{S M}+\mathcal{L}_{(t, b)}^{L E-t B E S S}=i \bar{\psi} \not \partial \psi-e \bar{\psi} A Q \psi-\frac{G_{L E}}{2} \bar{\psi} Z \\
& \left(C_{L} P_{L}+C_{R} P_{R}\right) \psi-\frac{g_{L E}}{\sqrt{2}} \bar{\psi}\left(W^{+} \tau^{+}+W^{-} \tau^{-}\right) \\
& \left(D_{L} P_{L}+D_{R} P_{R}\right) \psi
\end{aligned}
$$

where $\tau^{ \pm}=\tau^{1} \pm i \tau^{2}, P_{L, R}=\left(1 \mp \gamma_{5}\right) / 2$. For the light fermions (all SM fermions except the top and bottom quarks) $D_{L}=1, D_{R}=0$, and

$$
C_{L}=2 T_{L}^{3}-2 \kappa s_{\theta}^{2} Q, C R=-2 \kappa s_{\theta}^{2} Q,
$$

where

$$
\kappa=\frac{1+2 x^{2}}{1+4 s_{\theta}^{2} x^{2}},
$$

and

$$
\begin{aligned}
& g_{L E}^{2}=\frac{1+4 s_{\theta}^{2} x^{2}}{1+x^{2}} \frac{e^{2}}{s_{\theta}^{2}}, G_{L E}^{2}=\frac{\left(1+4 s_{\theta}^{2} x^{2}\right)^{2}}{c_{\theta}^{2}+x^{2}} \frac{e^{2}}{s_{\theta}^{2}} \\
& e=\frac{g g^{\prime} / G}{\sqrt{1+\left(\frac{g g^{\prime}}{G g^{\prime \prime} / 2}\right)^{2}}}, s_{\theta}=g^{\prime} / G, x=g / g^{\prime \prime},
\end{aligned}
$$

where $G=\left(g^{2}+g^{\prime 2}\right)^{1 / 2}$ and $c_{\theta}=\left(1-s_{\theta}^{2}\right)^{1 / 2}$. In the case of the top and bottom quarks

$$
\begin{aligned}
& C_{L}=2(1-\Delta L / 2) T_{L}^{3}-2 \kappa s_{\theta}^{2} Q, \\
& C_{R}=2\left(P_{f} \Delta R / 2\right) T_{R}^{3}-2 \kappa s_{\theta}^{2} Q,
\end{aligned}
$$

where $P_{t}=1, P_{b}=p^{2}$, and

$$
D_{L}=1-\Delta L / 2, D_{R}=p \Delta R / 2,
$$

where

$$
\Delta L=b_{L}-2 \lambda_{L}, \Delta R=b_{R}-2 \lambda_{R}
$$

Hence, the number of free parameters was reduced also in the fermion sector of the low-energy Lagrangian. The low-energy observables will depend on the combinations (13) of $b$ and $\lambda$ parameters only. Therefore, no limits derived from the low-energy measurements can apply to $b$ 's and $\lambda$ 's individually.

The deviations of the LE Lagrangian from its SM counterpart modify predictions for the low-energy observables. Thus we can use their measured values to derive the preferences and restrictions on the LE free parameters.

In particular, the experimental limits for the LE-tBESS parameters were derived by fitting the low-energy (pseudo) observables $\mathcal{E}_{1}, \mathcal{\varepsilon}_{2}, \mathcal{\varepsilon}_{3}, \Gamma_{b}(Z \rightarrow b \bar{b})$, and $B R\left(B \rightarrow X_{s} \gamma\right)$. The epsilons are related to the basic observables [13]: the ratio of the electroweak gauge boson masses, $r_{M} \equiv M_{W} / M_{Z}$; the inclusive partial decay width of $Z$ to the charged leptons, $\Gamma_{l}(Z \rightarrow l \bar{l}+$ photons $)$; the forward-backward asymmetry of charged leptons at the $Z$-pole, $A^{F B .}\left(M_{Z}\right)$; and the inclusive partial decay width of $Z$ to bottom quarks, $\Gamma_{b}(Z \rightarrow b \bar{b}+X)$.

The full-scale analysis along with its results can be found in [11]. By fitting the five observables mentioned above with the four free parameters $-x, \Delta L, \Delta R$, and $p-$ we found the best values

$$
g^{\prime \prime}(x)=29, \quad \Delta L=-0.004, \quad p \Delta R=0.003,
$$

with $x_{\text {min }}^{2}($ chi $)=2.40$. Since d.o.f. $=5-4=1$ the obtained value of $x^{2}{ }_{\text {min }}$ corresponds to the backing of $12 \%$. Within the rounding errors these values hold for the cut-off scale between $0.3 \mathrm{TeV} \leq \Lambda \leq 10^{3} \mathrm{TeV}$, at least. The best values of $p$ and $\Delta R$ depend on $\Lambda$, separately; in particular,

$$
\begin{aligned}
& \Lambda=1 \mathrm{TeV}: \quad \Delta R=0.016 \quad p=0.209 \\
& \Lambda=2 \mathrm{TeV}: \quad \Delta R=0.011 \quad p=0.289
\end{aligned}
$$

In Fig. 1 the $x^{2}$ values for various combinations of fixed $g$ " and $p$ are shown. We can see that the best backing for the fits is getting less pronounced as $g$ " approaches 30 from below. More specifically, while backings of the fits with different $p$ 's can differ by several orders of magnitude when $g$ " $<20$, the backing for $g$ " $=30$ changes between $10 \%$ and $50 \%$ as $p$ crawls along the $\langle 0 ; 1\rangle$ interval.

Recall that beside the direct interactions the new vector triplet couples to the third quark generation via mixing with the electroweak gauge bosons. In certain regions of the parameter space the negative interference between the two kinds of interactions takes place. Thus, it might happen that even though the direct couplings of the vector resonance to the top and/ 
or bottom quark are non-trivial the resonance will not decay through the given quark channel. Or, the particular decay will be suppressed below the value that would be implied by the indirect couplings alone. If this occurred the resonance peak in a process where $V$ decays to top and/or bottom quarks could disappear even though the resonance exists and couples directly to the third quark generation.

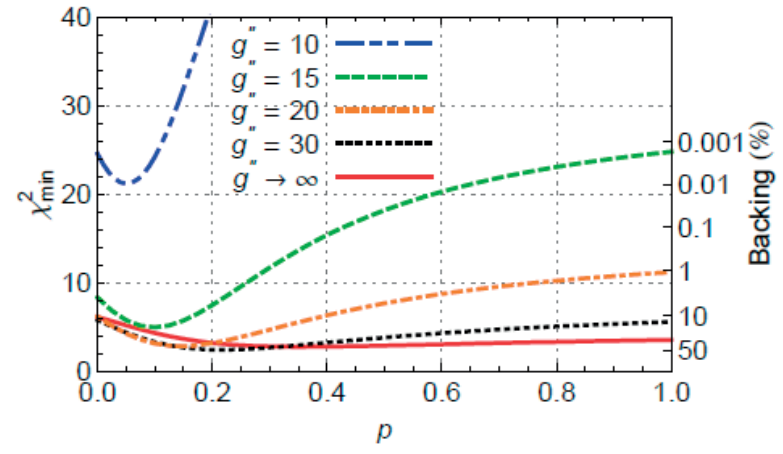

Fig. $1 x^{2}{ }_{\text {min }}$ of the fit by $\Delta L$ and $\Delta R$ as a function of the fixed parameters g" and p for $\Lambda=1 \mathrm{TeV}$; The labels on the r.h.s. axis indicate the backings for d.o.f. $=3$

We calculated the DV regions for $M_{V}=1 \mathrm{TeV}$ and for the parameter values $g$ " $=15$ (30) and $p=0.10$ (22). For each case, the DV's in three decay channels of the vector resonance triplet were found. The channels are $V^{ \pm} \rightarrow \bar{t} \bar{b} / \bar{t} b, V^{0} \rightarrow b \bar{b}$, and $V^{0} \rightarrow \bar{t} t$.

In Fig. 2, the DV regions of the three decay channels at $g "=15$ and $p=0.1$ are depicted. In each of the three graphs, there are the $95 \%$ C.L. electroweak precision data (EWPD) contours for $\Lambda=1 \mathrm{TeV}$ superimposed. The low-energy limits apply to the combination of $b$ 's and $\lambda$ 's $(\Delta L$ and $\Delta R$ ) rather than to the parameters alone. The low-energy limits depicted in the $b_{R}-b_{L}$

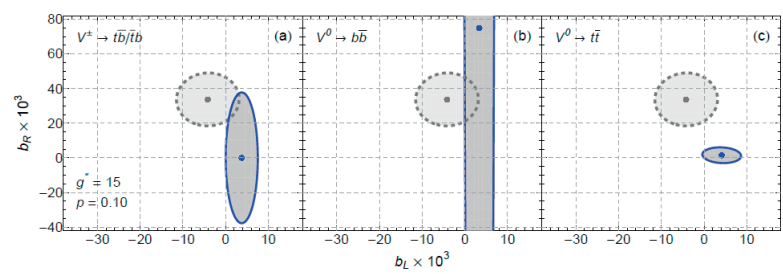

Fig. 2 The DV regions (dark-shaded areas with the blue solid boundary) of the $V$ vector resonance for three decay channels: (a) $V^{ \pm} \rightarrow t \bar{b} / \bar{t} b$,

(b) $V^{0} \rightarrow b \bar{b}$, and (c) $V^{0} \rightarrow t \bar{t}$. The blue dots inside the DV's indicate the point of no decay for the particular channels. The DV regions are calculated for $g "=15$ and $p=0.10$. The corresponding

95\% C.L. EWPD contours for $\Lambda=1 \mathrm{TeV}$ and $\lambda_{L}=\lambda_{R}=0$ are superimposed to the graphs as the regions with the gray dashed boundaries. The gray dots inside the EWPD regions indicate the point with the highest backing. Note that the $D V$ for the $V^{0} \rightarrow b \bar{b}$ channel exceeds the displayed range of the $b_{R}$ axis. The complete $D V$ region has an oval shape centered at the blue dot. Its lower and upper ends are found at $b_{R}=-0.272$ and $b_{R}=0.422$, respectively. graphs correspond to $\lambda_{L}=\lambda_{R}=0$. Nevertheless, by choosing nonzero values for $\lambda_{L, R}$ the low-energy contours get shifted around the $\left(b_{L}, b_{R}\right)$ parameter space. Various values of $\lambda$ 's can result either in no overlap of the low-energy regions with the DV's or in maximal overlap of the two areas.

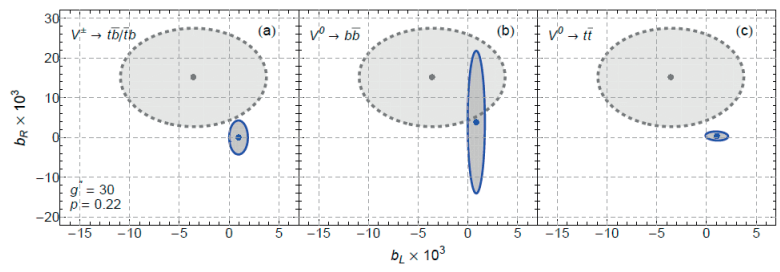

Fig. 3 The same as in Fig. 2 except for different values of $g$ ", and $p$. In this case, $g "=30$ and $p=0.22$

Figure 3 displays the same contents as Fig. 2, except for different values of the parameters $g$ " and $p$. Here, the values of the parameters correspond to $g$ " $=30$ and $p=0.22$. There are also the 95\% C.L. electroweak precision data contours for $\Lambda=1 \mathrm{TeV}$ and $\lambda_{L}=\lambda_{R}=0$ superimposed in Fig. 3 .

As we can see in Figs. 2 and 3 the DV areas are more or less comparable in size with the EWPD regions. In addition, both structures are located not far away from each other when $\lambda_{L}=\lambda_{R}=0$. Hence, there are reasonable values of the $\lambda$ parameters for which the significant part of an EPWD region falls inside the DV.

There might be new physics materialized through the existence of the new vector resonances as well as non-zero values of the $b$ parameters, yet it does not have to reveal itself in an experiment. If the actual values of the $b$ parameters fell in the DV it would make the detection and study of the new vector resonance more difficult. In particular, thanks to the indirect mixing-induced coupling the vector resonance can be produced and studied in the Drell-Yan processes at the LHC or in the s-channel at a future electron-positron collider [14 and 15]. As we demonstrated in [9] the signal of the vector resonance in the top and bottom decay channels can be diminished or hidden by the negative interference between the direct and indirect couplings.

\section{Conclusions}

We formulated and studied the effective Lagrangian for description of phenomenology of new scalar and vector resonances which might result from new strong physics beyond the SM. Following the often used and studied approach, the ESB sector of the effective Lagrangian was based on the $S U(2)_{L} \times S U(2)_{R} \rightarrow S U(2)_{L+R}$ non-linear sigma model while the scalar resonance was introduced as the $S U(2)_{L+R}$ singlet and identified with the newly discovered $125-\mathrm{GeV}$ boson. The vector resonance was built in as the $L+R$ triplet employing the hidden local symmetry approach. Throughout the paper we assumed the 
vector resonance mass at the bottom of the $\mathrm{TeV}$ scale. No other non-SM fields were considered in our effective Lagrangian.

There might be new physics materialized through the existence of the new vector resonances as well as non-zero values of the $b$ parameters, yet it does not have to reveal itself in an experiment. Even though there are no direct interactions of the vector resonance triplet to the light fermions the resonance does couple to the light fermions thanks to the mixing with the electroweak gauge bosons. This enables processes with a direct production of the vector resonance at the LHC and future electron-positron colliders. However, in the top and bottom decay channels the signal of the vector resonance can be diminished or hidden by the negative interference between the direct and indirect couplings. We calculated the regions of the negative interference for the studied values of the top-BESS parameters and found that they are often comparable in size and close in position with the 95\% C.L. regions. As a general tendency, the relative size of the DV's with respect to the low-energy allowed areas shrinks as the value of g" grows.

\section{Acknowledgments}

We would like to thank Ivan Melo for useful discussions. The work of M.G. and J.J. was supported by the Research Program MSM6840770029 and by the project International Cooperation ATLAS-CERN of the Ministry of Education, Youth and Sports of the Czech Republic. M.G. was supported by the Slovak CERN Fund. J.J. was supported by the NSP grant of the Slovak Republic.

\section{References}

[1] AAD, G. et al.: (ATLAS Collaboration), Phys. Lett. B 716, 1, 2012; Chatrchyan, S. et al. (CMS Collaboration), ibid. 30, 2012.

[2] ELLIS, J., You, T.: J. High Energy Phys. 09, 2012, 123.

[3] GIARDINO, P. P. , KANNIKE, K., RAIDAL, M., STRUMIA, A.: Phys. Lett. B 718, 469, 2012; Carmi, D., Falkowski, A., Kuflik, E., Volansky, T., Zupan, J.: J. High Energy Phys. 10, 2012, 196; Montull, M., Riva, F. ibid. 11, 2012, 018; Plehn, T., Rauch, M.: Europhys. Lett. 100, 11002, 2012; Corbett, T., Eboli, O. J. P., Gonzalez-Fraile, J., Gonzalez-Garcia, M. C.: Phys. Rev. D 86, 075013, 2012; arXiv:1211.4580; Peskin, M. E. arXiv:1208.5152.

[4] WeINBERG, S.: Phys. Rev. D 19, 1277, 1979; Susskind, L. ibid. 20, 2619, 1979; Farhi, E., Susskind, L.: Phys. Rept. 74, $277,1981$.

[5] DIMOPOUlOS, S., SUSSKIND, L.: Nucl. Phys. B155, 237, 1979; Eichten, E., Lane, K. D.: Phys. Lett. B90, 125, 1980.

[6] HOLdOM, B.: Phys. Rev. D 24, 1441, 1981; Phys. Lett. B150, 301, 1985; Yamawaki, K., Bando, M., Matumoto, K.-i.: Phys. Rev. Lett. 56, 1335, 1986; Appelquist, T., Karabali, D., Wijewardhana, L. C. R.: ibid. 57, 957, 1986; Akiba, T., Yanagida, T.: Phys. Lett. B169, 432, 1986; Appelquist, T., Wijewardhana, L. C. R.: Phys. Rev. D 36, 568, 1987; Lane, K., Eichten, E.: Phys. Lett. B222, 2741989.

[7] HILL, C. T.: Phys. Lett. B266, 419, 1991; B345, 483, 1995.

[8] BANDO, M., KUGO, T., YAMAWAKI, K.: Phys. Rep. K. 217, 1988.

[9] GINTNER, M., JURAN, J., MELO, I.: Phys. Rev. D 84, 035013, 2011.

[10] GINTNER, M., Juran, J.: arXiv:1301.2124.

[11] GINTNER, M., JURAN, J.: Europ. Phys. J. C73 (2013) 2577.

[12] The D0 Collaboration, Phys. Lett. B713, 2012, 165-171.

[13] Altarelli, G., BARBIERI, R., CARAVAGlios, F.: Nucl. Phys. B405, 3, 1993; Int. J. Mod. Phys. A13, 1031, 1998.

[14] ACCOMANDO, E., BECCIOLINI, D., DE CURTIS, S., DOMINICI, D., FEDELI, L.: Phys. Rev. D 83, 115021, 2011.

[15] ACCOMAndo, E., DE CURTIS, S., DOMINICI, D., FEDELI, L.: Phys. Rev. D 79, 055020, 2009; Gintner, M., Melo, I., Trpisova, B.: arXiv:0903.1981; Barducci, D., Belyaev, A., De Curtis, S., Moretti, S., Pruna, G. M.: arXiv:1210.2927. 